\title{
Elbow Scattering and Boundary Value Problems of NLPDE
}

Pierre C SABATIER

Laboratoire de Physique Mathématique et Théorique CNRS -UMR 5825

Université de Montpellier II, 34095 Montpellier Cedex 5, France

\begin{abstract}
We use Lax equations to define a scattering problem on an infinite elbow shaped line of the $(x, t)$ plane. The evolution of scattering coefficients when the elbow is translated in the plane shows how convenient scannings may reconstruct the solution $V(x, t)$ of the nonlinear equation associated to the Lax pair. It also helps us understanding why this can work only if strong consistency conditions, related to the asymptotic behaviors of $V$, are satisfied by the boundary values.
\end{abstract}

\section{Introduction to elbow scattering}

Let $x, t \in \mathbb{R}$. We call $t=t_{0}$ an $x$-path, $x=x_{0}$ a $t$-path. Let $F(k, x, t)$ be a 2 -vector continous solution of the equations

$$
\begin{aligned}
\frac{\partial}{\partial x} F(k, x, t) & =\mathbf{M}(k, x, t) F(k, x, t), \\
\frac{\partial}{\partial t} F(k, x, t) & =\mathbf{N}(k, x, t) F(k, x, t),
\end{aligned}
$$

where $\mathbf{M}$ and $\mathbf{N}$ are $2 \times 2$ matrices, with zero trace, depending continously on $k, x$ and $t$. We call "elbow" a path which is half space and half time: hereafter, more precisely, the elbow $\mathcal{E}\left(P_{0}\right)$ is the path

$$
\mathcal{E}\left(P_{0}\right)=\left\{x, t \mid\left(t=t_{0}, x \geq x_{0}\right),\left(x=x_{0}, t \geq t_{0}\right)\right\}
$$

whose apex $P_{0}$ coordinates are $x_{0}, t_{0}$.

Thanks to the zero trace assumption, the determinant of the matrix made out of two columns which are everywhere continuous solutions of (1.1) and (1.2) is an invariant along the elbow (and throughout any bounded connected domain in $\mathbb{R} \times \mathbb{R}$ ). $F$ and $G$ are two independent solutions if $\operatorname{det}[F, G] \neq 0$, and then any solution $H$ of (1.1) and (1.2) is a linear combination of $F$ and $G$, whose coefficients (complex numbers) only depend on $k$.

Suppose now that $\mathbf{M}$ and $\mathbf{N}$ enable us to define:

a) two independent "time Jost solutions", $\vec{G}^{ \pm}(k, 0, t)$, respectively asymptotic to $e_{1}^{ \pm}(k, t)$ as $t \rightarrow \infty$ on the $x=0$ axis, and $k \in \mathbb{R} \backslash\{0\}$.

b) two independent "space Jost solutions", $\overleftarrow{G}^{ \pm}(k, x, 0)$, respectively asymptotic to $e_{0}^{ \pm}(k, x)$ as $x \rightarrow \infty$ on the $t=0$ axis, and $k \in \mathbb{R} \backslash\{0\}$. 
The scattering problem on the elbow $\mathcal{E}(0)$ is settled by the linear relations between these couples of Jost solutions, and the "scattering coefficients" are calculated from their determinants.

Let us translate the elbow into $\mathcal{E}\left(P_{0}\right)$ and suppose that the solutions $\vec{G}^{ \pm}$and $\overleftarrow{G}^{ \pm}$ of (1.1), (1.2) can be uniquely continued into $\overrightarrow{\mathbf{G}}^{ \pm}\left(k, x_{0}, t\right)$ and $\overleftarrow{\mathbf{G}}^{ \pm}\left(k, x, t_{0}\right)$. A restandardisation recovering the adequate asymptotic behavior enables us to derive from the Jost solutions on $\mathcal{E}\left(P_{0}\right)$, say $\overrightarrow{\mathbf{G}}^{ \pm}\left(k, x_{0}, t\right)$ and $\overleftarrow{\mathbf{G}}^{ \pm}\left(k, x, t_{0}\right)$. If it can be done simply, there is a simple relation between the scattering problem on $\mathcal{E}\left(P_{0}\right)$ and that on $\mathcal{E}(0)$. Such a result may give a simple way $[1,2]$ to study the evolution of the consistency relation between (1.1) and (1.2) throughout the quarterplane $x \geq 0, t \geq 0$.

\section{The KdV case}

Let us set

$$
\mathbf{M}=\mathbf{M}_{0}+\mathbf{V}, \quad \mathbf{N}=\mathbf{N}_{0}+\mathbf{W}, \quad \mathbf{N}_{0}=k^{2} \mathbf{M}_{0},
$$

and

$$
\begin{aligned}
& \mathbf{M}_{0}=\left(\begin{array}{cc}
0 & 1 \\
-k^{2} & 0
\end{array}\right), \quad \mathbf{V}=\left(\begin{array}{cc}
0 & 0 \\
V(x, t) & 0
\end{array}\right), \\
& \mathbf{W}=\left(\begin{array}{cc}
V_{1} & V_{0} \\
k^{2} V_{0}+V_{2} & -V_{1}
\end{array}\right), \\
& V_{0}=\frac{1}{2} V(x, t), \quad V_{1}=-\frac{1}{4} V^{\prime}, \quad V_{2}=\frac{1}{2} V^{2}-\frac{1}{4} V^{\prime \prime} .
\end{aligned}
$$

In these formulas, prime denotes the derivative with respect to $x$. Now, it is readily seen that if $F(k, x, t)$ has continuous second derivatives (as a function of $x$ and $t$ ), the consistency condition between (1.1) and (1.2) reads

$$
\frac{\partial \mathbf{M}}{\partial t}-\frac{\partial \mathbf{N}}{\partial x}+[\mathbf{M}, \mathbf{N}]=0
$$

which holds if and only if the "Korteveg de Vries" equation (KdV) holds:

$$
\frac{\partial V}{\partial t}+\frac{1}{4} V^{\prime \prime \prime}-\frac{3}{2} V V^{\prime}=0
$$

Thus, a solution $F \in C^{2}$ yields a solution $V$ of KdV. Now, if $V$ and the two first derivatives go to zero rapidly enough as $x$ or $t$ goes to $\infty$, Jost solutions do exist, with the asymptotic behaviors:

$$
e_{0}^{ \pm}(k, x)=\left(\begin{array}{c}
\mp i \\
k
\end{array}\right) e^{ \pm i k x} ; e_{1}^{ \pm}(k, t)=\left(\begin{array}{c}
\mp i \\
k
\end{array}\right) e^{ \pm i k^{3} t}
$$

If $V, V^{\prime}, V^{\prime \prime}$ are bounded for finite $x, t$, equation (2.1) and (2.2) can be transformed into Volterra integral equations and the continuations $\overleftarrow{\mathbf{G}}^{ \pm}$and $\overrightarrow{\mathbf{G}}^{ \pm}$do exist too. On the elbow $\mathcal{E}(0)$, the "elbow scattering coefficients" $\underset{c}{\rightleftarrows}$ and $\underset{d}{\rightleftarrows}$ are defined by the matchings:

$$
\overleftarrow{G}^{\mp}(k, x, t)=\overleftarrow{c}^{ \pm}(k) \vec{G}^{\mp}(k, x, t)+\overleftarrow{d}^{ \pm}(k) \vec{G}^{ \pm}(k, x, t)
$$




$$
\vec{G}^{\mp}(k, x, t)=\vec{c}^{ \pm}(k) \overleftarrow{G}^{\mp}(k, x, t)+\vec{d}^{ \pm}(k) \overleftarrow{G}^{ \pm}(k, x, t)
$$

They are easily derived from the values of $\operatorname{det}\left[\overleftarrow{G}^{\mp}, \vec{G}^{ \pm}\right]$and $\operatorname{det}\left[\overleftarrow{G}^{\mp}, \vec{G}^{\mp}\right]$ at the point 0 of $\mathcal{E}(0)$, where $\vec{G}^{ \pm}$and $\overleftarrow{G}^{ \pm}$are both defined. Because the determinant of two solutions of (1.1) and (1.2) is invariant, the formulas (2.8) and (2.9) hold true if $\vec{G}^{ \pm}$and $\overleftarrow{G}^{ \pm}$are replaced by their continuations $\overrightarrow{\mathbf{G}}^{ \pm}$and $\overleftarrow{\mathbf{G}}^{ \pm}$, solving (1.1) and (1.2), everywhere these continuations hold. It is easy to standardize $\overleftarrow{\mathbf{G}}^{ \pm}$, respectively $\overrightarrow{\mathbf{G}}^{ \pm}$, into a Jost solution $\overleftarrow{G}^{ \pm}$on the axis $t=t_{0}, x \geq 0$, respectively into a Jost solution $\overrightarrow{\mathbf{G}}^{ \pm}$on the axis $x=x_{0}$ $t \geq 0$ : one should only follow respectively the path $\left(\infty, 0 \rightarrow t_{0}\right)$, respectively $\left(0 \rightarrow x_{0}, \infty\right)$, and use Eqs. (1.1) and (1.2), in order to derive the asymptotic behavior of $\overleftarrow{\mathbf{G}}^{ \pm}$or $\overrightarrow{\mathbf{G}}^{ \pm}$, which is that of the zero potential solution:

$$
E^{ \pm}(k, x, t)=\left(\begin{array}{c}
\mp i \\
k
\end{array}\right) \exp \left[ \pm i\left(k x+k^{3} t\right)\right]
$$

Hence we get:

$$
\begin{aligned}
& \vec{G}^{ \pm}\left(k, x_{0}, t\right)=\exp \left[\mp i k x_{0}\right] \overrightarrow{\mathbf{G}}^{ \pm}\left(k, x_{0}, t\right) \\
& \overleftarrow{G}^{ \pm}\left(k, x, t_{0}\right)=\exp \left[\mp i k t_{0}\right] \overleftarrow{\mathbf{G}}^{ \pm}\left(k, x, t_{0}\right)
\end{aligned}
$$

It follows that if we go from the elbow $\mathcal{E}(0)$ to the elbow $\mathcal{E}\left(P_{0}\right)$, the new scattering coefficients are equal to those of (2.8) and (2.9) times factors $e^{ \pm i k x_{0}}, e^{ \pm i k^{3} t_{0}}$. If we knew $V\left(x, t_{0}\right)$ tending to zero also as $x \rightarrow-\infty$ so that a Jost solution $g^{ \pm}$could be defined by its asymptotic behavior there, and if we knew the reflection coefficient on the $x$-axis, $t=0$, formulas similar to (2.12) would reproduce the well-known inverse scattering factor to $R^{+}\left(k, t_{0}\right)$ as $t$ goes from 0 to $t_{0}$. Similar results would be obtained if $x$ and $t$ are interchanged. But in the present paper, we stick at the quarter plane $x \geq 0, t \geq 0$. To see what can be done, we introduce now what we call the boundary scattering coefficients:

$$
\begin{aligned}
& \stackrel{\rightleftarrows}{a}+(k, x, t)=(2 i k)^{-1} \operatorname{det}\left[E^{-}, \overleftrightarrow{\mathbf{G}}^{+}\right](k, x, t), \\
& \stackrel{\rightleftarrows}{b}+(k, x, t)=-(2 i k)^{-1} \operatorname{det}\left[E^{-}, \overleftrightarrow{\mathbf{G}}^{-}\right](k, x, t) .
\end{aligned}
$$

Suppose we are given in a "consistent" way the values $V(x, 0)$ for all $x \geq 0, V(0, t)$, $V^{\prime}(0, t), V^{\prime \prime}(0, t)$ for all $t \geq 0$. It is possible to calculate $\overrightarrow{\mathbf{G}}^{ \pm}$on the $t$ half axis, $\overleftarrow{\mathbf{G}}^{ \pm}$on the $x$ half axis, hence the $\mathcal{E}(0)$ scattering coefficients at $x=0$. Then, using the coefficients and Eqs. (2.8) and (2.9), we can derive $\overleftarrow{\mathbf{G}}^{ \pm}$on the $t$ half-axis and $\overrightarrow{\mathbf{G}}^{ \pm}$on the $x$ half axis of the elbow. Hence we can derive $\underset{a}{\vec{a}}+$ and $\overleftrightarrow{b}^{+}$for $(k, 0, t)$ and for $(k, x, 0)$. Now suppose that, at the point $\left(0, t_{0}\right)$, we match the Jost solution that corresponds to a zero potential on the half axis $\left(x \leq 0, t_{0}\right)$ with the Jost solutions $\overleftarrow{G}^{ \pm}\left(k, 0, t_{0}\right)$

$$
\left(\begin{array}{c}
i \\
k
\end{array}\right)=\overleftarrow{a}_{t_{0}}^{+}(k) \overleftarrow{G}^{-}\left(k, 0, t_{0}\right)+\overleftarrow{b}_{t_{0}}^{+}(k) \overleftarrow{G}^{+}\left(k, 0, t_{0}\right)
$$

The matching of $E^{-}$with $\overleftarrow{\mathbf{G}}^{-}$and $\overleftarrow{\mathbf{G}}^{+}$can be written with the coefficients $\overleftarrow{a}^{+}\left(k, 0, t_{0}\right)$ and $\overleftarrow{b}^{+}\left(k, 0, t_{0}\right)$. Comparing with (2.15) after using (2.12) yields

$$
\overleftarrow{a}_{t_{0}}^{+}(k)=\overleftarrow{a}^{+}\left(k, 0, t_{0}\right), \quad \overleftarrow{b}_{t_{0}}^{+}(k)=e^{2 i k^{3} t_{0}} \overleftarrow{b}^{+}\left(k, 0, t_{0}\right)
$$


Equation (2.15) represents a Schrödinger scattering "on the line" by the potential $\theta(x) V\left(x, t_{0}\right)$ (where $\theta$ is the Heaviside function). The transmission and reflection coefficients $\left(\overleftarrow{a}_{t_{0}}^{+}\right)^{-1}$ and $\overleftarrow{b}_{t_{0}}^{+} / \overleftarrow{a}_{t_{0}}^{+}$enable us to derive $V\left(x, t_{0}\right)$ for any $x \geq 0$ by using the Faddeev-Marchenko method. Hence, scanning the quarter plane $x \geq 0, t \geq 0$ by axes $t=t_{0}$ enables one reconstructing $V(x, t)$. But a similar scanning can be done by axes $x=x_{0}$. On each half axis $x=x_{0}$, the scattering coefficients can be derived from $\vec{a}^{+}\left(k, x_{0}, 0\right)$ and $\vec{b}^{+}\left(k, x_{0}, 0\right)$ and a method recently devised by the author [3] enables one reconstructing $V\left(x_{0}, t\right)$. It is clear that the consistency is implied only if special conditions hold on the datas $V(x, t), x \geq 0$ and $\left\{V(0, t), V^{\prime}(0, t), V^{\prime \prime}(0, t\}, t \geq 0\right.$.

\section{Consistency conditions}

We assume that datas are real and that we are seeking a real function $V(x, t)$. It is easy to show from (1.1), (1.2), (2.1), (2.2), that $\overleftarrow{G}^{+}$and $\overleftarrow{G}^{-}$, respectively $\vec{G}^{+}$and $\vec{G}^{-}$, are complex conjugate of each other. This fact can be used for deriving, say, $\overleftarrow{\mathbf{G}}_{1}^{ \pm}$and $\overleftarrow{\mathbf{G}}_{2}^{ \pm}$, from $\operatorname{det}\left[E^{-}, \overleftarrow{\mathbf{G}}^{+}\right]$and $\operatorname{det}\left[E^{-}, \overleftarrow{\mathbf{G}}^{-}\right]$. It is also easy to see that if these two determinants are $C^{2}$ functions of $x$ and $t$, so do $\overleftarrow{\mathbf{G}}^{+}$and $\overleftarrow{\mathbf{G}}^{-}$, and the reverse is obvious. Hence, it follows from Eqs. (2.13) and (2.14) that $\mathrm{KdV}$ is equivalent to a $C^{2}$ condition on both $\overleftarrow{a}^{+}$ and $\overleftarrow{b}^{+}$. Going further is simplified by two formulas, which follow from Eqs. (1.1), (1.2) and the zero trace property:

$$
\begin{aligned}
\frac{\partial}{\partial x} \operatorname{det}\left[E^{-}, \overleftarrow{\mathbf{G}}^{ \pm}\right] & =-\operatorname{det}\left[\mathbf{V} E^{-}, \overleftarrow{\mathbf{G}}^{ \pm}\right] \\
\frac{\partial}{\partial t} \operatorname{det}\left[E^{-}, \overleftarrow{\mathbf{G}}^{ \pm}\right] & =-\operatorname{det}\left[\mathbf{W} E^{-}, \overleftarrow{\mathbf{G}}^{ \pm}\right]
\end{aligned}
$$

Writing down that $\frac{\partial^{2}}{\partial x \partial t} b^{+}=\frac{\partial^{2}}{\partial t \partial x} b^{+}$gives with the help of Eqs. (3.1) and (3.2) the consistency formula

$$
\frac{\partial}{\partial t}\left[V e^{-i z} \overleftarrow{\mathbf{G}}_{1}^{-}\right]=\frac{\partial}{\partial x}\left[\left(\omega_{2} \overleftarrow{\mathbf{G}}_{1}^{-}-\omega_{1} \overleftarrow{\mathbf{G}}_{2}^{-}\right) e^{-i z}\right]
$$

where

$$
\omega_{1}=V_{1}-i k V_{0}, \quad \omega_{2}=V_{2}+i k V_{1}+k^{2} V_{0}, \quad z=k x+k^{3} t
$$

and the same treatment of $a^{+}$yields

$$
\frac{\partial}{\partial t}\left[V e^{-i z} \overleftarrow{\mathbf{G}}_{1}^{+}\right]=\frac{\partial}{\partial x}\left[\left(\omega_{2} \overleftarrow{\mathbf{G}}_{1}^{+}-\omega_{1} \overleftarrow{\mathbf{G}}_{2}^{+}\right) e^{-i z}\right]
$$

Similar formulas could be obtained for $\overrightarrow{\mathbf{G}}$, but we shall mainly play on the $x$-scanning. Similar formulas can also be obtained for the "linearized" problem, where (1.1) and (1.2) are replaced by

$$
\frac{\partial}{\partial x} F^{ \pm}-\mathbf{M}_{0} F^{ \pm}-\mathbf{V} E^{ \pm}=0=\frac{\partial}{\partial t} F^{ \pm}-\mathbf{N}_{0} F^{ \pm}-\mathbf{W}^{0} E^{ \pm}
$$

$\mathbf{W}^{0}$ being $\mathbf{W}$ except for the replacement of $V_{2}$ by $V_{2}^{0}=-\frac{1}{4} \frac{\partial^{2} V}{\partial x^{2}}$ and $\mathrm{KdV}$ is replaced by $\mathrm{LKdV}$, which is $\mathrm{KdV}$ without the nonlinear term, and is also the consistency formula for a $C^{2}$ solution of (3.7):

$$
\frac{\partial V}{\partial t}+\frac{1}{4} \frac{\partial^{3} V}{\partial x^{3}}=0
$$


the treatment of boundary scattering coefficients yields a formula analogous to (3.3):

$$
\frac{\partial}{\partial t}\left[V e^{-2 i z}\right]=\frac{\partial}{\partial x}\left[\left(\omega_{2}^{0}+i k \omega_{1}\right) e^{-2 i z}\right]
$$

whereas the formula analogous to (3.6) reduces to (3.8). If $V$ and derivatives are such that $\Omega^{0}=\omega_{2}^{0}+i k \omega_{1}$ goes to zero as $x$ goes to $\infty$, integrating (3.8) yields the evolution of the Fourier transform of $V(x, t) \theta(x)$ :

$$
\frac{\partial}{\partial t} \int_{0}^{\infty} V(x, t) e^{-2 i\left(k x+k^{3} t\right)} d x=-\Omega^{0}(k, 0, t) e^{-2 i k^{3} t},
$$

where the right hand side is known from data. The value of the left hand side may give the desired result but if we require for example that $\int_{0}^{\infty}|V(x, t)| d x \rightarrow 0$ as $t \rightarrow \infty$, the data must satisfy the coupling consistency condition:

$$
\int_{0}^{\infty} V(x, 0) e^{-2 i k x} d x=\int_{0}^{\infty} \Omega^{0}(k, 0, t) e^{-2 i k^{3} t} d t
$$

Other sort of requirements on asymptotic behaviors are associated to other conditions, and classes of data which are sufficient to guarantee that the scanning methods do work have not very simple characterizations [1].

Let us come back to the nonlinear case, with (3.3) and (3.6).

If $V, V^{\prime}, V^{\prime \prime}$ go to zero conveniently as $x \rightarrow \infty$, we obtain from (3.3) and (3.6) formulas analogous to (3.10):

$$
\frac{\partial}{\partial t} \int_{0}^{\infty} V(x, t) e^{-i z} \overleftarrow{\mathbf{G}}_{1}^{\mp}(k, x, t) d x=\left[\omega_{1} \overleftarrow{\mathbf{G}}_{2}^{\mp}-\omega_{2} \overleftarrow{\mathbf{G}}_{1}^{\mp}\right](k, 0, t) e^{-i k^{3} t}
$$

which give the evolution of the boundary scattering coefficients along with the scanning and may give the desired result. Again, if we assume that $\int_{0}^{\infty}|V(x, t)| d t$ vanishes at $t \rightarrow \infty$, we derive a point of necessary coupling consistency conditions between values which are derivable on the $x=0$ and $t=0$ half axes:

$$
\int_{0}^{\infty} V(x, 0) e^{-i k x} \overleftarrow{G}_{1}^{\mp}(k, x, 0) d x=\int_{0}^{\infty}\left[\omega_{2} \overleftarrow{\mathbf{G}}_{1}^{ \pm}-\omega_{1} \overleftarrow{\mathbf{G}}_{2}^{\mp}\right](k, 0, t) e^{-i k^{3} t} d t
$$

As in the linear case, ansatz (using an IST representation of Jost solutions) can give classes of data sufficient to solve the problem, but not simple.

\section{References}

[1] Sabatier P C, Elbow Scattering and Inverse Scattering. Applications to LKdV and KdV, J. Math. Phys., 2000, V.41, 414-436

(to be published).

[2] Pelloni B and Fokas T, A Spectral Approach for Linear and Integrable Nonlinear PDE's in Arbitrary Domains, Preprint.

[3] Sabatier P C, New Direct Linearizations for KdV and Solutions of the Other Cauchy Problem, J. Math. Phys., 1999, V.40, 2983-3020. 